\title{
Manifestações extraesofágicas da doença do refluxo gastroesofágico
}

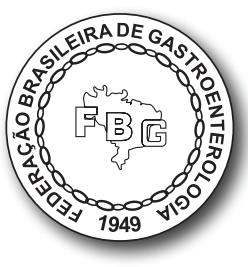

Eponina M. O. Lemme

\section{INTRODUÇÃO}

A doença do refluxo gastroesofágico (DRGE) é uma doença de alta prevalência na população em geral. Estima-se que aproximadamente 7 a 10\% dos indivíduos saudáveis apresentem azia, o sintoma principal da doença diariamente ${ }^{1}$.

Estudo populacional no Brasil, avaliando cerca de 14 mil indivíduos saudáveis, demonstrou que em torno de $12 \%$ apresentavam azia pelo menos 1 vez por semana ${ }^{2}$.

Em 2006, um grupo de especialistas reunido em Montreal estabeleceu uma definição de DRGE baseada em evidências, como "sintomas incômodos causados pelo refluxo do conteúdo gástrico para o esôfago", ocorrendo de forma moderada 2 vezes por semana ou de forma intensa pelo menos 1 vez por semana ${ }^{3}$.

O grupo classificou a DRGE em síndromes esofágicas (sintomáticas e com lesões, como esofagite, estenose e esôfago de Barrett) e síndromes extraesofágicas (SEE). Estas são divididas em SEE de associação estabelecida e de associação proposta (Quadro 1). Neste capítulo, serão abordadas as SEE de associação estabelecida, chamadas de manifestações extraesofágicas (MEE).

Quadro 1 Síndromes extraesofágicas

$\begin{array}{ll}\text { Associação estabelecida } & \text { Associação proposta } \\ \text { - Tosse } & \cdot \text { Faringite } \\ \text { - Laringite } & \cdot \text { Sinusite } \\ \text { - Asma } & \cdot \text { Fibrose pulmonar crônica } \\ \text { - Erosão dentária } & \cdot \text { Otite média }\end{array}$

Fonte: adaptado de Vakil et al., $2006^{3}$. 


\section{FISIOPATOLOGIA DAS MANIFESTAÇÕES EXTRAESOFÁGICAS}

O mecanismo fisiopatológico pelo qual o refluxo gastroesofágico (RGE) induz as MEE não está, ainda, muito bem elucidado. Admitem-se dois mecanismos para o surgimento dessas manifestações:

- A teoria do refluxo, segundo a qual o RGE atingiria a faringe/laringe, ocasionando lesões nestas áreas por contato (manifestação otorrinolaringológica - ORL), ou seria aspirado, gerando broncoespasmo/infecção (manifestação respiratória).

- A teoria do reflexo, em que o estímulo pelo ácido refluído geraria um reflexo de natureza vagal, desencadeando tosse e pigarro, os quais, acentuados pelo hábito e por fatores emocionais, propiciariam lesões na laringe e perpetuação dos sintomas. O mesmo estímulo poderia gerar reflexo esôfago-brônquico, com vias aferente e eferente de natureza vagal e surgimento de broncoespasmo. A combinação dos dois mecanismos poderia também estar presente no mesmo paciente ${ }^{1}$.

\section{MANIFESTAÇÕES RESPIRATÓRIAS}

Das manifestações respiratórias associadas ao refluxo, a asma e a tosse crônica são as mais estudadas.

Há maior incidência de refluxo em asmáticos, sendo encontrado em 55 a $80 \%$ dos pacientes, porém é difícil avaliar a verdadeira importância da associação, pois tanto a DRGE como a asma têm grande prevalência na população em geral. Pacientes com DRGE têm maior prevalência de asma do que os não refluidores, sendo o fator isolado que mais dificulta o controle da asma ${ }^{1}$.

O surgimento de sintomas respiratórios na idade adulta, a piora ou o desencadeamento dos sintomas com refeições copiosas, ingestão de bebidas alcoólicas ou durante a madrugada são elementos para o diagnóstico. Outras peculiaridades seriam a ausência de componente intrínseco e a má resposta a broncodilatadores e corticosteroides. Quando interrogados, 40 a 70\% dos pacientes apresentam sintomas típicos, existindo portanto uma parcela de pacientes em que a asma pode ser a única manifestação.

Considera-se tosse crônica a que persiste por 8 ou mais semanas em não fumantes ${ }^{1}$. Está presente em 10 a $40 \%$ dos pacientes com refluxo e a maioria atribui seu início a uma infecção respiratória.

A tríade principal da tosse crônica envolve asma, secreção pós-nasal e refluxo. A tosse relacionada a refluxo predomina durante o dia e em posição ortostática, durante a fonação, ao se levantar da cama e ao se alimentar. Alguns pacientes podem relatar sintomas noturnos. Pode ser não produtiva ou produ- 
zir excesso de muco, tendo duração média de 13 a 58 meses. Entre 40 e 70\% dos pacientes não têm sintomas de refluxo, enquanto a parcela restante relata os sintomas típicos precedendo ou sucedendo o aparecimento da tosse.

Os critérios de seleção para investigar refluxo em pacientes com tosse crônica incluem não tabagistas, ausência de irritantes ambientais, exclusão de asma, secreção pós-nasal e uso de inibidores de enzima conversora de angiotensina (ECA), radiografia de tórax e seios da face normais.

Outras manifestações que podem estar relacionadas ao refluxo são pneumonia de aspiração, bronquiectasia, bronquite crônica, fibrose pulmonar e esclerose sistêmica progressiva.

\section{MANIFESTAÇÕES OTORRINOLARINGOLÓGICAS}

A relação entre DRGE e manifestações do trato aerodigestivo superior ou ORL também tem sido bastante estudada. Cerca de $25 \%$ dos pacientes com DRGE apresentam sintomas relacionados a cabeça e pescoço e 10\% dos pacientes que procuram o otorrinolaringologista têm sintomas ou sinais que podem ser atribuídos a refluxo. As queixas mais frequentes são rouquidão, redução do volume ou falha vocal, pigarro, necessidade de clareamento da garganta e sensação de globus cervical, sintomas que surgem isoladamente ou em combinação. Observam-se disfagia de localização alta, por vezes com engasgos ao ato da deglutição, e sensação de asfixia noturna, acompanhada ou não de estridor laríngeo. Metade dos pacientes, quando interrogada, apresentará queixas típicas e a existência de pirose diária ou frequente é preditiva de refluxo em pacientes com queixas ORL ${ }^{4}$. É importante uma história cuidadosa, pois as queixas laríngeas necessitam de diagnóstico diferencial. Fatores de risco associados incluem uso frequente da voz, tabagismo, história de infecções do trato respiratório superior, vômitos, secreção pós-nasal, regurgitação noturna, sintomas alérgicos, contato com animais, uso recente de ar condicionado ou exposição a ambientes novos contendo material sintético. As manifestações ORL mais frequentes estão descritas no Quadro 2.

\begin{tabular}{l} 
Quadro 2 Manifestações otorrinolaringológicas da doença do refluxo gastroesofágico \\
\hline - Rouquidão, alterações vocais \\
\hline - Tosse, pigarro, globo \\
\hline - Dor de garganta \\
\hline - Faringite, laringite \\
\hline - Granuloma de prega vocal \\
\hline - Estenose subglótica \\
\hline - Câncer de laringe \\
Fonte: adaptado de Richter JE, 20051.
\end{tabular}


Recentes "candidatos" a manifestações ORL da DRGE são os pacientes com sinusite crônica, otite média recorrente, causa frequente de otite em crianças, e laringoespasmo paroxístico ${ }^{5}$.

Deve-se suspeitar de refluxo em casos de rouquidão pela manhã, piora dos sintomas com chocolate, café, refeições copiosas, ganho de peso precedendo o início dos sintomas e presença de pirose e/ou regurgitação ácida, observada em metade dos pacientes ${ }^{1}$.

A maioria dos pacientes com queixa ORL, tenha ou não sintomas típicos associados, procura um otorrinolaringologista para investigação, sendo na maioria das vezes realizada laringoscopia. Anormalidades na laringe associadas à DRGE incluem laringite posterior (edema ou eritema), frequentemente referida como "laringite de refluxo", nódulos em pregas vocais, úlceras de contato e granulomas, estenose de laringe, cobblestoning de parede posterior e muco excessivo. No entanto, essas anormalidades não são específicas da DRGE. Um estudo demonstrou pelo menos um desses achados em $85 \%$ de um grupo de mais de 200 voluntários saudáveis ${ }^{6}$.

\section{INVESTIGAÇÃO}

Infelizmente, não há um teste definitivo que identifique o refluxo como causa das manifestações extraesofágicas da DRGE.

A investigação deve se iniciar com a realização da endoscopia digestiva alta (EDA), embora a esofagite erosiva seja incomum nesse grupo de pacientes aproximadamente 5 a 10\%, de modo geral leve, ao contrário das manifestações típicas, em que está presente em 30 a $50 \%$ destes ${ }^{1}$. Alguns recomendam a EDA apenas em pacientes com queixas típicas associadas de longa duração e para a procura de esôfago de Barrett.

$\mathrm{Na}$ ausência de esofagite erosiva, o método de escolha para o diagnóstico de refluxo nesse grupo de pacientes é a pHmetria esofágica prolongada ( $\mathrm{PHM}$ ), que apresenta maior sensibilidade neste particular. Metanálise envolvendo 5 estudos e 316 pacientes com queixas ORL demonstrou refluxo anormal em $44 \%$, em média ${ }^{7}$. Em pacientes asmáticos, a metanálise de nove estudos e 646 pacientes encontrou média de refluxo anormal por PHM em $51 \%{ }^{8}$. Outra metanálise demonstrou PHM anormal em 53\% de 1.145 pacientes com laringite, em $66 \%$ de 504 asmáticos e em $89 \%$ de 126 pacientes com tosse crônica ${ }^{9}$.

A positividade da PHM depende em grande parte dos critérios de seleção (história altamente sugestiva, pacientes consecutivos ou ao acaso), do número de pacientes e da natureza do estudo (prospectivo, retrospectivo). Um estudo prospectivo de nosso Serviço, envolvendo 52 pacientes selecionados com tosse 
crônica, 65\% mulheres e média de idade de 55 anos, demonstrou pHmetria alterada em $33 \%$ deles $^{10}$.

$\mathrm{Na}$ investigação das manifestações extraesofágicas da DRGE, há alguns anos foi preconizada a PHM de dois sensores, o distal na posição tradicional (5 $\mathrm{cm}$ acima do esfíncter inferior, como determinado pela esofagomanometria EMN) e um proximal. Entretanto, não existe consenso a respeito da importância do refluxo proximal, dos valores normais e nem do melhor posicionamento do segundo sensor, se no esôfago alto, no esfíncter superior ou na faringe. Falsos positivos também foram descritos. Em decorrência dessas controvérsias, tem sido questionada a utilidade da PHM de dois sensores ${ }^{3}$.

\section{QUAL O PAPEL DO REFLUXO "NÃO ÁCIDO" NAS MEE DA DRGE?}

O refluxo "não ácido" ou fracamente ácido é o que ocorre no período pós-prandial e o refluxato é o conteúdo gástrico tamponado pelo alimento ou cujo $\mathrm{pH}$ foi alterado pelo uso de inibidor de bomba de prótons (IBP). Tem sido relacionado a sintomas persistentes, principalmente à tosse e à regurgitação, em pacientes em uso de IBP. O refluxo "não ácido" pode ser demonstrado pelo emprego da impedâncio-pHmetria prolongada (IMP-pH), equipamento que avalia, por meio de cateter contendo pares de eletrodos de impedância, o trânsito de bolus no interior do esôfago, quer de forma anterógrada (deglutição, alimento) ou retrógrada (refluxo) e sua consistência (líquida, sólida ou gasosa). $\mathrm{O} \mathrm{pH}$ do refluxato é avaliado por um sensor de $\mathrm{pH}$, podendo ser ácido $(\mathrm{pH}$ abaixo de 4) ou "não ácido" ou fracamente ácido (acima de 4). A maior importância do método é a possibilidade de correlação entre os sintomas ocorridos e episódios de refluxo (índice de sintomas positivo), sejam eles ácidos ou não ácidos, para que se possa estabelecer o tratamento adequado ${ }^{11}$.

Um estudo de 100 pacientes com tosse crônica submetidos a IMP-pH revevou refluxo ácido anormal em $45 \%$, ausência de refluxo (ácido e não ácido) em $31 \%$ e refluxo não ácido anormal em $24 \%$, sendo que em $17 \%$ destes o índice de sintomas foi positivo, ou seja, houve coincidência do sintoma tosse com episódio de refluxo não ácido ${ }^{11}$. Estima-se também que 12 a 15\% dos pacientes com tosse crônica persistente na vigência de IBP possam ter este sintoma relacionado a refluxo "não ácido"12.

\section{NOVAS TECNOLOGIAS}

Recentemente foi proposta a utilização de cateter nasofaríngeo (RESTECH), pouco invasivo, na avaliação das MEE da DRGE. Um estudo preliminar sugere 
benefícios em pacientes com suspeita de refluxo faringolaríngeo, sendo preditor de bons resultados cirúrgicos em pacientes com MEE quando comparados à $\mathrm{pHmetria}$ proximal ${ }^{13}$. Estudos controlados são necessários para se avaliar a real utilidade do método.

\section{MEE E DRGE: associação ou causalidade?}

Interroga-se se as manifestações extraesofágicas da DRGE seriam uma associação ou causalidade, decorrente de sua elevada prevalência. Há significativa associação epidemiológica entre as MEE e DRGE, inclusive com explicações fisiopatológicas plausíveis. Um estudo prospectivo desenhado para tentar responder a esta questão avaliou 39 asmáticos adultos, submetidos a provas de função respiratória com broncodilatadores, EDA, esofagomanometria e PHM, além de questionário orientado para queixas esofágicas. Refluxo anormal à PHM foi observado em $51 \%$ dos pacientes. Em análise multivariada, o único fator preditivo de refluxo foi a existência de pirose frequente, ou seja, 2 ou mais vezes por semana ${ }^{14}$. Outro estudo prospectivo de metodologia semelhante avaliou 54 pacientes com queixas ORL, empregando laringoscopia, EDA, EMN e PHM. Refluxo anormal foi observado em $37 \%$ dos pacientes, sendo a pirose frequente o único fator preditivo ${ }^{4}$.

Portanto, se a associação entre MEE e DRGE pode ser sugerida pelo encontro de esofagite, PHM anormal e pirose frequente, a causalidade ainda é objeto de especulação. Um estudo avaliou 140 pacientes com tosse crônica submetidos a tratamento com IBP ou fundoplicatura. Obtiveram melhora significativa da tosse $61 \%$ dos pacientes. O fatores preditivos de resposta foram a presença de sintomas típicos frequentes, PHM anormal com \% de tempo de $\mathrm{pH}<4 \geq$ $12 \%$ e a presença de hérnia hiatal $\geq 4 \mathrm{~cm}^{15}$.

\section{TRATAMENTO DAS MANIFESTAÇÕES EXTRAESOFÁGICAS}

Se refluxo anormal pode ser encontrado em inúmeros pacientes portadores de MEE, a resposta ao tratamento da DRGE é menos evidente e inconstante.

Em relação à asma, revisão de oito estudos controlados e randomizados ${ }^{16}$ demonstrou melhora sintomática de $60 \%$ dos asmáticos tratados e reduziu a necessidade de uso de broncodilatadores, porém a melhora das provas de função pulmonar (PFP) ocorreu em apenas $25 \%$ dos pacientes. Outra metanálise envolvendo 12 estudos de pacientes tratados com antagonistas H2 ou IBP demonstrou que, de modo geral, não havia melhora das PFP, dos sintomas da asma ou noturnos, embora um seleto subgrupo tenha se beneficiado da terapêutica antirrefluxo ${ }^{17}$. 
Estudo recente de pacientes com tosse crônica, randomizado, placebo-controlado, com IBP dose dupla, demonstrou ausência de melhora dos sintomas ou da qualidade de vida dos pacientes ${ }^{18}$. Metanálise envolvendo oito estudos controlados (IBP vs. placebo) e 344 pacientes com laringite crônica não mostrou diferença na redução dos sintomas ${ }^{19}$. Estudo controlado empregando esomeprazol 2 vezes/dia $v s$. placebo por 4 meses em 145 pacientes com "laringite de refluxo" revelou que a resolução dos sintomas ORL ocorreu em apenas 15 e 16\% dos dois grupos sem diferença significativa ${ }^{20}$. Outro estudo controlado envolvendo um pequeno número de pacientes (22) com laringite idiopática obteve $50 \%$ de resposta sintomática significativa ao lanzoprazol 2 vezes/dia comparado ao placebo (17\%) por 3 meses $^{21}$.

Em nosso serviço, 18 pacientes com tosse crônica e refluxo anormal à PHM foram sumetidos a placebo ou a pantoprazol dose dupla por 3 meses, sendo o desfecho a redução de escore de tosse. Houve sucesso em $60 \%$ do grupo pantoprazol e em 37,5\% do grupo placebo, porém as diferenças não foram significativas, talvez pelo pequeno número de pacientes ${ }^{10}$.

As razões da falta de resposta das MEE ao tratamento com IBP têm sido analisadas e envolvem falta de adesão ao tratamento, necessidade de doses maiores do que as habituais, além de tempo mais prolongado de tratamento. $\mathrm{Na}$ maior parte dos casos, o paciente melhora da pirose, preditora da associação, e persistem as MEE, sugerindo que elas não seriam causadas por RGE.

Questiona-se se o tratamento deve ser feito na base da suspeita clínica (estratégia empírica) ou após estratégia investigativa, com o diagnóstico de refluxo anormal feito por PHM antes do tratamento. Nas duas estratégias, preconiza-se, além das medidas posturais e comportamentais, o emprego de IBP 2 vezes/dia (jejum e antes do jantar) por 3 meses, avaliando-se a resposta e/ou prosseguimento de investigação posteriormente ${ }^{22}$.

\section{TRATAMENTO DO REFLUXO "NÃO ÁCIDO"}

Como referido, o refluxo "não ácido" ou fracamente ácido tem sido relacionado à tosse crônica, notadamente naqueles que não respondem adequadamente ao tratamento com IBP. O tratamento do refluxo "não ácido" envolve uso de inibidores dos relaxamentos transitórios do esfíncter esofágico inferior, como o baclofeno, que é um antagonista do ácido gama-aminobutírico. A dose inicial é de $5 \mathrm{mg}$, 3 vezes/dia, com aumento progressivamente até $20 \mathrm{mg}, 3$ vezes/dia. Entretanto, os efeitos colaterais como tonturas, náuseas e sonolência limitam seu uso ${ }^{23}$.

O encaminhamento de pacientes com MEE para cirurgia antirrefluxo por meio de fundoplicatura laparoscópica deve ser criterioso. Faltam estudos con- 
trolados e deve-se analisar caso a caso e principalmente o impacto da persistência do sintoma na qualidade de vida do paciente. Os resultados não são tão expressivos como naqueles operados por sintomas típicos. Refluxo ácido anormal ou índice de sintomas positivo em exame prévio sem IBP têm sido observados como preditivos de sucesso da fundoplicatura ${ }^{23}$.

\section{CONCLUSÕES}

As MEE estão presentes em 30 a 60\% dos portadores de DRGE. Metade apresentará queixas típicas que sugerem DRGE. Atenção para associação de patologias que podem confundir o quadro clínico. Resposta satisfatória ao tratamento com IBP sugere causalidade. Um grupo de pacientes poderá ter sintomas associados a refluxo não ácido. As indicações cirúrgicas devem ser criteriosas.

\section{REFERÊNCIAS BIBLIOGRÁFICAS}

1. Richter J. Extraesophageal manifestations of GERD. Alim Pharmacol Ther. 2005;11(suppl):70-80.

2. Moraes-Filho JP, Chinzon D, Eisig JN, et al. Prevalence of heartburn and Gastroesophageal reflux disease in the urban Brazilian population. Arq Gastroenterol. 2003;42:122-7

3. Vakil N, van Zanten SV, Kahrilas P, et al. The Montreal definition and classification of GERD: a global evidence based consensus. Am J Gastroenterol. 2006;101:1900-20

4. Souza A, Lemme E. Manifestações otorrinolaringológicas e doença do refluxo gastroesofágico Boletim da FBG, edição especial, 1998, p. 76-9.

5. Poelmans J, Tack J. Supraesophageal manifestations of gastro-oesophageal reflux disease. Gut. 2005;54:1492-99.

6. Hicks DM, Ours TM, Abelson TI, et al. The prevalence of hypopharinx findings associated with GERD in normal volunteers. J Voice. 2002;16:564-79.

7. Wong RK, Col M, Waring P, et al. ENT manifestations of gastroesophageal reflux. Am J Gastroenterol. 2000;95:S15-S22

8. Haveman BD, Henderson CA, El-Serag HB. The association between gastro-oesophageal reflux disease and asthma: a systematic review. Gut. 2007;56:1654-64.

9. Vaezi MF.Review article: the role of $\mathrm{pH}$ monitoring in extraoesophageal gastro-oesophageal reflux disease. Aliment Pharmacol Ther. 2006;23Suppl1:40-9.

10. Rottenberg CL. Tosse crônica e doença do refluxo gastroesofágico: associação e causalidade. Tese de Mestrado em Gastroenterologia. Rio de Janeiro, UFRJ, julho/2012.

11. Blondeau K, Dupont LJ, Mertens V, et al. Improved diagnosis of gastro-oesophageal reflux in patients with unexplained chronic cough. Aliment Pharmacol Ther. 2007;25:723-32.

12. Patterson N, Mainie I, Rafferty G, et al. Nonacid reflux episodes reaching the pharynx are important factors associated with cough. J Clin Gastroenterol. 2009;43:414-9.

13. Worrell SG, DeMeester SR, Greene CL, et al. Pharyngeal pH monitoring better predicts a successful outcome for extraesophageal reflux symptoms after antireflux surgery. Surg Endosc. 2013;27:4113-8.

14. Nascentes R, Lemme E, Santos MIV. Does gastroesophageal reflux disease play a role in asthma?Am J Crit Care Med. 155(4):A967. 
15. Naik RD, Vaezi MF. Extra-esophageal manifestations of GERD: who responds to GERD therapy?Curr Gastroenterol Rep. 2013;15:318.

16. Field SK, Sutherland LR. Does medical anti-reflux therapy improve asthma in asthmatics with GERD? A critical review of the literature. Chest. 1998;14:275-83.

17. Coughlan JL, Gibson PG, Henry RJ. Medical treatment for reflux esophagitis does not consistently improve asthma control: a systematic review. Thorax. 2001;56:198-04.

18. Shaheen NJ, Crokett SD, Bright SD, et al. Randomized clinical trial : high-dose acid suppression for chronic cough - a double-blind, placebo-controlled study. Aliment Pharmacol Ther. 2011;33:225-34.

19. Qadeer MA, Phillips CO, Lopez AR, et al. Proton pump inhibitor therapy for suspected GERD-related chronic laryngitis: a meta-analysis of randomized controlled trials. Am J Gastroenterol. 2006;101:2646-54.

20. Vaezi MF, Richter JE, Stasney CR, et al. Treatment of chronic posterior laryngitis with esomeprazole. Laryngoscope. 2006;116:254-60

21. El-Serag HB, Lee P, Buchner A, et al. Lansoprazole treatment of patients with chronic idiopathic laryngitis: a placebo-controlled trial. Am J Gastroenterol. 2001;96:979-83.

22. Galmiche JP, Zerbib F, des Varannes B. Review article: respiratory manifestations of gastro-oesophageal reflux disease. Alim Pharm Ther. 2008;17:449-64.

23. Sifrim D, Zerbib F.Diagnosis and management of patients with reflux symptoms refractory to proton pump inhibitors. Gut. 2012;61:1340-54. 